\title{
A case of tubulointerstitial nephritis and uveitis syndrome after suspected drug-induced acute interstitial nephritis
}

\author{
Yukiko Kitamura $^{1}$, shohei kuraoka ${ }^{1}$, Koji Nagano ${ }^{1}$, and hiroshi tamura ${ }^{1}$ \\ ${ }^{1}$ Kumamoto University
}

December 22, 2021

\begin{abstract}
Distinguishing between late-onset TINU syndrome and drug-induced AIN remains difficult given that patients with TINU syndrome may develop uveitis long after the onset of AIN. Therefore, ophthalmic examination is required not only upon diagnosis but also continuously or when eye symptoms, and relapse of urinary findings are observed.
\end{abstract}

A case of tubulointerstitial nephritis and uveitis syndrome after suspected drug-induced acute interstitial nephritis

Yukiko Kitamura, Shohei Kuraoka, Koji Nagano, Hiroshi Tamura

Department of Pediatrics, Faculty of Life Sciences, Kumamoto University, Kumamoto, Japan, 1-1-1 Honjo, Kumamoto 860-8556, Japan.

Short title: TINU syndrome after suspected drug-induced AIN

Correspondence: Hiroshi Tamura

Department of Pediatrics, Faculty of Life Sciences, Kumamoto University, Kumamoto, Japan, 1-1-1 Honjo, Kumamoto 860-8556, Japan.

E-mail:bohm1905HT@kuh.kumamoto-u.ac.jp

Abstract

Distinguishing between late-onset TINU syndrome and drug-induced AIN remains difficult given that patients with TINU syndrome may develop uveitis long after the onset of AIN. Therefore, ophthalmic examination is required not only upon diagnosis but also continuously or when eye symptoms, and relapse of urinary findings are observed.

\section{Key words}

Tubulointerstitial nephritis and uveitis, drug-induced acute interstitial nephritis, DLST

\section{Introduction}

Tubulointerstitial nephritis and uveitis (TINU) syndrome, which is characterized by a combination of acute interstitial nephritis (AIN) and uveitis, is predominant among adolescent girls. Uveitis and pyelonephritis appear at various timepoints, with studies suggesting that more than half of the patients develop ocular symptoms later than AIN [1]. Therefore, early disease recognition may be challenging. This report also describes the reporting and diagnostic problems of TINU cases in female patients whose renal features are primarily due to drug-induced AIN. 


\section{Case}

A 15-year-old female patient was admitted to our hospital due to progressive deterioration of her renal function with an increased serum creatinine $(\mathrm{sCr})$ level of $0.95 \mathrm{mg} / \mathrm{dL}$ despite having no past history of renal disease. Two weeks prior to admission, she developed a fever $>38{ }^{\circ} \mathrm{C}$ and was empirically treated with azithromycin combined with the occasional use of acetaminophen (ACP) by a local physician; however, her fever persisted. Meanwhile, she started to complain of fatigue and nausea, which prompted referral to our hospital for further workup. Her medical history was unremarkable, except for her prior treatment with escitalopram for developmental disability for 6 months. Upon admission, the patient had a weight of $47 \mathrm{~kg}$ (1 $\mathrm{kg}$ weight loss), height of $149 \mathrm{~cm}$, temperature of $36.6{ }^{\circ} \mathrm{C}$, pulse rate of $90 \mathrm{beats} / \mathrm{min}$, and a blood pressure of 108/65 mmHg. Her bowel sounds were normal. Her abdomen was soft and flat, with lower abdominal tenderness, without rebound pain or palpable masses. The lungs were clear, and heart sounds were normal. No rash, lymphadenopathy, petechiae, or joint swelling were observed. Renal sonography showed no abnormal findings.

Diffusion-weighted images on abdominal magnetic resonance imaging showed heterogeneous high signals in both renal parenchyma and renal enlargement, which were consistent with acute tubular interstitial nephritis. No abnormalities in the fundus were observed. Aside from renal dysfunction, a serological study revealed an increased C-reactive protein (CRP) level $(4.24 \mathrm{mg} / \mathrm{dL})$ and negative findings for antineutrophil cytoplasmic antibody, antiglomerular basement membrane antibodies, anti-SSA/Ro antibodies, and antiSSB/La antibodies. Urinalysis showed active sediments with six white blood cells and three red blood cells per high-power field. Her urinary protein creatinine ratio was $0.4 \mathrm{~g} / \mathrm{g}$ [?]Cr. The urinary $\beta 2$-microglobulin (b2MG)/creatinine ratio and $N$-acetyl-beta-d-glucosaminidase (NAG)/creatinine ratio were $3.6 \mu \mathrm{g} / \mathrm{mg}[?] \mathrm{Cr}$ (reference range: $<0.3 \mu \mathrm{g} / \mathrm{mg}[$ ?]Cr) and $33.1 \mathrm{U} / \mathrm{g}[$ ?]Cr (reference range: $1.6-5.8 \mathrm{U} / \mathrm{g}[$ ?]Cr), respectively. A drug lymphocyte stimulation test (DLST) confirmed that the patient had a negative stimulation index (SI) score for ACP but had specific high SI scores for escitalopram of $432 \%$ (cutoff value for DLST positivity, $180 \%)$. Based on the lack of findings suggesting ocular disease following ophthalmological screening and laboratory results, as well as her clinical history, drug-induced AIN was suspected. The oral administration of escitalopram was stopped. A week after admission, the patient was then found to have anterior bilateral uveitis without any apparent abnormalities of the fundus oculi during a subsequent ophthalmological review. Thereafter, a renal biopsy was performed on the following day. Under a light microscope, five glomeruli were identified within the renal parenchyma. The glomerular structures were preserved, with marked diffuse infiltration of lymphocytes and some eosinophils accompanied by partial destruction of the tubular structures (Figure 1). Immunohistochemistry did not observe any immune complex deposits among the observed glomeruli. A diagnosis of TINU syndrome was thereafter established, and the patient was started on oral prednisolone (PSL) $60 \mathrm{mg}$ daily $(1.3 \mathrm{mg} / \mathrm{kg} /$ day), to which a favorable response was noted. The patient's ocular symptoms decreased 1 week after steroid administration, and her urinary b2MG/creatinine ratio and NAG/creatinine ratio decreased to $2.0 \mu \mathrm{g} / \mathrm{mg}[?] \mathrm{Cr}$ and $1.8 \mathrm{U} / \mathrm{g}[?] \mathrm{Cr}$, respectively. PSL was tapered to $45 \mathrm{mg} /$ day for 2 weeks. Relapse of uveitis was observed during steroid dose reduction, for which steroid instillation was started. Given that her eye symptoms could be controlled with steroid eye drops, the dose of the steroid was gradually tapered and discontinued 3 months after the start of steroid administration. No recurrence was observed as of 1 year after the discontinuation. The patient's clinical course is detailed in Figure 2.

\section{Discussion}

Establishing a clinical diagnosis of drug-induced AIN is exceedingly difficult given the numerous types of medications associated with AIN. Although AIN often occurs less than 2 weeks after drug exposure, it has been shown to vary from days to months depending on the drug [2]. However, a diagnosis of AIN may be established through knowledge of the patient's medical history and specific laboratory tests, such as DLST [2-5]. In fact, DLST has been regarded as a useful diagnostic test for various types of drug hypersensitivity, including drug-induced AIN, by measuring drug-induced T cell proliferation in vitro [6,7]. In the current case, considering that the patient showed a specific high score for Lexaburo in DLST, the 
patient's renal abnormality was initially considered to have been attributed to drug-induced AIN. We believe that the subsequent development of TINU syndrome in our patient may have been caused by the additive, synergistic, or cumulative effects of the drugs, including Lexaburo. Although DLST results are not absolute, $\mathrm{T}$ cell-mediated mechanisms have been proposed to play a major pathogenic role in TINU syndrome, which necessitates drug induction [2,7]. Case information on prior drug use as an estimated risk for TINU syndrome continues to accumulate [7-10]. However, similar inducers have been identified in both isolated AIN and TINU syndromes $[2,7,8]$. Unfortunately, determining the exact contribution of each is quite difficult. Whether AIN without TINU syndrome and uveitis is a different phenotype of the same pathology is particularly important and crucial issue that needs to be extensively investigated [11]. As such, we note that recent studies on the pathogenic role of modified CRP (mCRP) can irreversibly dissociate from native CRP in TINU syndrome $[12,13]$. This is because it can be one of the common target autoantigens in kidney and eye tissue $[10,13,14]$. Interestingly, serum levels of anti-mCRP autoantibodies may play a role in distinguishing patients with TINU syndrome from those with AIN $[13,14]$. Unfortunately, such a procedure is inapplicable to the current patient. Moreover, HLA-type analysis is progressing in TINU syndrome, with reports showing that HLADQA1, HLA-DQB1, and HLA-DRB1 are associated with the onset $[11,15]$. The HLA type in the current case was unknown, with the patient not consenting to genetic testing. Identifying the patient's mCRP and HLA types may have helped establish a diagnosis, and we believe that similar cases will benefit from such tests in the future. The main treatment for AIN is to discontinue or reduce the dose of the problematic drug $[2,4]$. Corticosteroid therapy has shown no clear benefits after discontinuation of the problematic drug. One study showed that $72 \%$ of patients with TINU syndrome who did not use systemic steroids were expected to spontaneously recover from renal dysfunction [16]. In contrast, another report revealed that two out of four patients who did not receive steroids progressed to chronic renal failure, with one patient developing end-stage renal failure [8]. Several reports have suggested corticosteroid administration may shorten the course of renal failure $[4,5,17]$. Considering that no improvement in the renal function of the current patient was observed following drug discontinuation, PSL was started at $60 \mathrm{mg} /$ day $(1.3 \mathrm{mg} / \mathrm{kg} /$ day). The interval between drug discontinuation and start of steroid treatment was approximately 3 weeks. Immediately after starting PSL treatment, the patient's renal function improved. This finding is consistent with that reported in previous studies, which showed that patients often respond to PSL doses of $1-2 \mathrm{mg} / \mathrm{kg} /$ day. Many authors agree with corticosteroid treatment for patients with TINU syndrome who have advanced renal failure similar to AIN. Simultaneous use of topical ocular steroids and immunosuppressants may be essential due to the prolonged and/or recurrent uveitis associated with this syndrome [18-20].

\section{Conclusion}

Distinguishing between late-onset TINU syndrome and drug-induced AIN remains difficult given that patients with TINU syndrome may develop uveitis long after the onset of AIN. Therefore, ophthalmic examination is required not only upon diagnosis but also continuously or when eye symptoms (e.g., photophobia and conjunctival suffusion), elevation of urinary $\beta 2 \mathrm{MG}$, and relapse of urinary findings (e.g., hematuria and proteinuria) are observed.

\section{Statements}

\section{Acknowledgments}

We would like to thank the patient for participating in this study.

\section{Statement of Ethics}

This case study was conducted ethically in accordance with the World Medical Association Declaration of Helsinki. The article is exempt from Kumamoto University Ethical Committee approval as a single case is not required for approval.

Consent to publish statement: Written informed consent for examinations and to publish their cases including images was obtained from our patient and her parents.

\section{Conflict of Interest Statement}


All authors declare that they have no conflict of interest relating to this case report.

\section{Funding Sources}

The authors received no financial support for the research, authorship, and/or publication of this article.

Author Contributions

K. Yukiko and T. Hiroshi cared for the patients and designed the project. N. Koji and K. Shohei collected clinical information. All authors have read and accepted the manuscript.

\section{Data Availability Statement}

All data generated or analysed during this study are included in this article. Further enquiries can be directed to the corresponding author.

\section{REFERENCES}

1. Mandeville JT, Levinson RD, Holland GN, et al. The tubulointerstitial nephritis and uveitis syndrome. Surv Ophtkalomol 2001; 46: 195-208

2. Praga M, Gonza lez E. acute interstitial nephritis. Kidney int. 2010; 77: 956-61

3. Rossert J. Drug-induced acute interstitial nephritis. Kidney Int . 2001;60(2):804-17.

4. Onishi A, Yamamoto H, Akimoto T, et al. Reversible acute renal failure associated with clomipramine-induced interstitial nephritis. Clin Exp Nephrol. 2007;11(3):241-3.

Akimoto T, Horikoshi R, Muto S, Kusano E. Low-dose corticosteroid and gallium-67 scintigraphy and acute interstitial nephritis. Saudi J Kidney Dis Transpl. 2014;25(4):864-8.

6. Pichler WJ, Tilch J. The lymphocyte transformation test in the diagnosis of drug

hypersensitivity. Allergy . 2004;59(8):809-20. 7. Suzuki H, Yoshioka K, Miyano M, et al. Tubulointerstitial nephritis and uveitis

(TINU) syndrome caused by the Chinese herb "Goreisan". Clin Exp Nephrol . 2009;13(1):73-6.

8. Cacoub P, Deray G, Le Hoang P, et al. Idiopathic acute interstitial nephritis associated with anterior uveitis in adults. Clin Nephrol . 1989;31(6):307-10. 9. Kindler J, Kemper R, Helmchen U. Acute tubulointerstitial nephritis and uveitis syndrome (TINU syndrome). Occurrence of uveitis after stopping steroids.

Nephrol Dial Transplant . 1998;13(7):1892-3. 10. Santoro D, Vita G, Rovito S, et al. Drug-induced TINU syndrome and genetic

characterization. Clin Nephrol . 2012;78(3):230-6.

11. Levinson RD, Park MS, Rikkers SM, et al. Strong associations between specific

HLA-DQ and HLA-DR alleles and the tubulointerstitial nephritis and uveitis syndrome. Invest Ophthalmol Vis Sci . 2003;44(2):653-7.

12. Tan Y, Yu F, Yang H, Chen M, Fang Q, Zhao MH. Autoantibodies against

monomeric C-reactive protein in sera from patients with lupus nephritis are asso- ciated with disease activity and renal tubulointerstitial lesions. Hum Immunol . 2008;69(12):840-4.

13. Tan Y, Yu F, Qu Z, et al. Modified C-reactive protein might be a target autoa- ntigen of TINU syndrome. Clin J Am Soc Nephrol . 2011;6(1):93-100. 
14. Li C, Su T, Chu R, Li X, Yang L. Tubulointerstitial nephritis with uveitis in Chinese adults. Clin J Am Soc Nephrol . 2014;9(1):21-8.

15. Perasaari J, Saarela V, Nikkila J, et al. HLA associations with tubulointerstitial nephritis with or without uveitis in Finnish pediatric population: nation-wide study. Tissue Antigens 2013; 81(6): 435-41.

16. matumoto $\mathrm{k}$, fukunari $\mathrm{k}$, ikeda $\mathrm{y}$, et al. a report of an adult case of tubulointerstitial nephritis and uveitis syndrome, with a review of 102 japanease cases. Am j case rep 2015; 16: 119-23

17. Toto RD. Acute tubulointerstitial nephritis. Am J Med Sci . 1990;299(6):392-410.

18. Mackie FE, Rosenberg AR, Kainer G. Tubulointerstitial nephritis: drugs are not

always to blame. J Paediatr Child Health . 2008;44(5):305-7. 19. Vohra S, Eddy A, Levin AV, Taylor G, Laxer RM. Tubulointerstitial nephritis and uveitis in children and adolescents. Four new cases and a review of the litera-

ture. Pediatr Nephrol . 1999;13(5):426-32. 20. Goda C, Kotake S, Ichiishi A, Namba K, Kitaichi N, Ohno S. Clinical features

in tubulointerstitial nephritis and uveitis (TINU) syndrome. Am J Ophthalmol .

2005;140(4):637-41.

Figure Legend

Figure 1.

There was no glomerular sclerosis, mesangial proliferative, membrane change.

Significant stromal cell infiltration and tubular atrophy were observed.

a, b: PAS staining, $\times 200$

Fig. 2 Creatine, urinary $\beta 2$-microglobulin, and the treatment during the observation period.
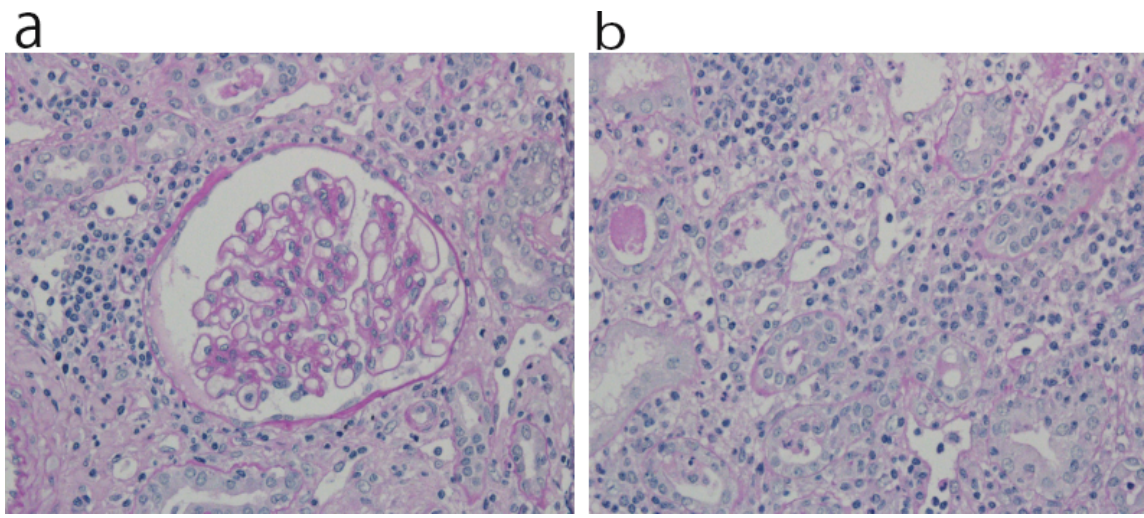
The clinical course

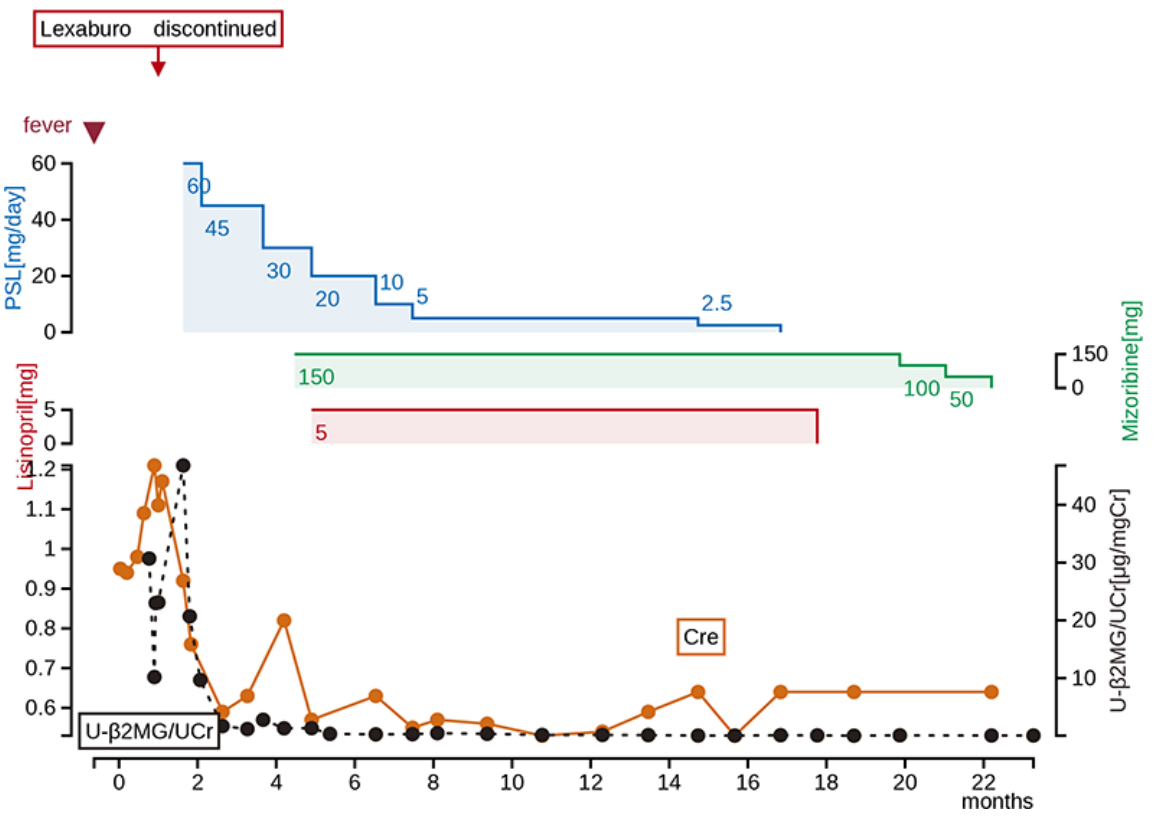

